\title{
Smooth Orthogonal Drawings of Planar Graphs
}

\author{
Md. Jawaherul Alam ${ }^{1}$, Michael A. Bekos ${ }^{2}$, Michael Kaufmann ${ }^{2}$, \\ Philipp Kindermann ${ }^{3}$, Stephen G. Kobourov ${ }^{1}$, and Alexander Wolff ${ }^{3}$ \\ ${ }^{1}$ Department of Computer Science, University of Arizona, USA \\ $\{$ mjalam, kobourov\}@cs.arizona.edu \\ ${ }^{2}$ Wilhelm-Schickhard-Institut für Informatik, Universität Tübingen, Germany \\ \{bekos, mk\}einformatik. uni-tuebingen. de \\ 3 Lehrstuhl für Informatik I, Universität Würzburg, Germany \\ http://www1.informatik.uni-wuerzburg.de/en/staff
}

\begin{abstract}
In smooth orthogonal layouts of planar graphs, every edge is an alternating sequence of axis-aligned segments and circular arcs with common axisaligned tangents. In this paper, we study the problem of finding smooth orthogonal layouts of low edge complexity, that is, with few segments per edge. We say that a graph has smooth complexity $k$-for short, an $\mathrm{SC}_{k}$-layout-if it admits a smooth orthogonal drawing of edge complexity at most $k$.

Our main result is that every 4-planar graph has an $\mathrm{SC}_{2}$-layout. While our drawings may have super-polynomial area, we show that, for 3-planar graphs, cubic area suffices. Further, we show that every biconnected 4-outerplane graph admits an $\mathrm{SC}_{1}$-layout. On the negative side, we demonstrate an infinite family of biconnected 4-planar graphs that requires exponential area for an $\mathrm{SC}_{1}$-layout. Finally, we present an infinite family of biconnected 4-planar graphs that does not admit an $\mathrm{SC}_{1}$-layout.
\end{abstract}

\section{Introduction}

In the visualization of technical networks such as the structure of VLSI chips [8] or UML diagrams [10] there is a strong tendency to draw edges as rectilinear paths. The problem of laying out networks in such a way is called orthogonal graph drawing and has been studied extensively. For drawings of (planar) graphs to be readable, special care is needed to keep the number of bends small. In a seminal work, Tamassia [11] showed that one can efficiently minimize the total number of bends in orthogonal layouts of embedded 4-planar graphs, that is, planar graphs of maximum degree 4 whose combinatorial embedding (the cyclic order of the edges around each vertex) is given. In contrast to this, minimizing the number of bends over all embeddings of a 4-planar graph is NP-hard [6].

In a so far unrelated line of research, circular-arc drawings of graphs have become a popular matter of research in the last few years. Inspired by American artist Mark Lombardi (1951-2000), Duncan et al. [4] introduced and studied Lombardi drawings, which are circular-arc drawings with the additional requirement of perfect angular resolution, that is, for each vertex, all pairs of consecutive edges form the same angle. Among others, Duncan et al. treat drawings of $d$-regular graphs where all vertices have 
to lie on one circle. They show that under this restriction, for some subclasses, Lombardi drawings can be constructed efficiently, whereas for the others, the problem is NP-hard. They also show [5] that trees can always be Lombardi drawn in polynomial area, whereas straight-line drawings with perfect resolution may need exponential area.

Very recently, Bekos et al. [2] introduced the smooth orthogonal graph layout problem that combines the two worlds; the rigidity and clarity of orthogonal layouts with the artistic style and aesthetic appeal of Lombardi drawings. Formally, a smooth orthogonal drawing of a graph is a drawing on the plane where (i) each vertex is drawn as a point; (ii) edges leave and enter vertices horizontally or vertically, (iii) each edge is drawn as an alternating sequence of axis-aligned line segments and circular-arc segments such that consecutive segments have a common horizontal or vertical tangent at their intersection point. In the case of (4-) planar graphs, it is additionally required that (iv) there are no edge-crossings. Note that, by construction, (smooth) orthogonal drawings of 4-planar graphs have angular resolution within a factor of two of optimal.

Figure 1 shows a real-world example: a smooth orthogonal drawing of an Austrian regional bus and train map. Extending our model, the map has (multi-) edges that enter vertices diagonally (as in Grünau im Almtal Postamt; bottom right).

For usability, it is important to keep the visual complexity of such drawings low. In a (smooth) orthogonal drawing, the complexity of an edge is the number of segments it consists of, that is, the number of inflection points plus one. Then, a natural optimization goal is to minimize, for a given (embedded) planar graph, the edge complexity of a drawing, which is defined as the maximum complexity over all edges.

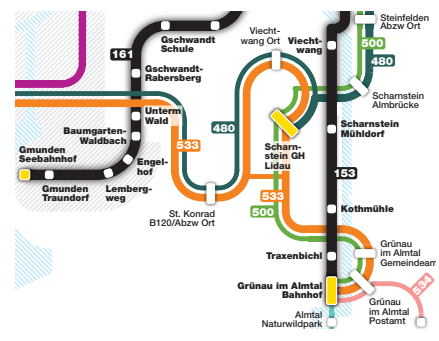

Fig. 1: Clipping of the public transport map Gmunden Vöcklabruck - Salzkammergut, Austria [1] We say that a graph has orthogonal complexity $k$ if it admits an orthogonal drawing of edge complexity at most $k$, for short, an $O C_{k}$-layout. Accordingly, we say that a graph has smooth complexity $k$ if it admits a smooth orthogonal drawing of edge complexity at most $k$, for short, an $S C_{k}$-layout. We seek for drawings of 4-planar graphs with low smooth complexity.

Our Contribution. Known results and our contributions to smooth orthogonal drawings are shown in Table 1 The main result of our paper is that any 4-planar graph admits an $\mathrm{SC}_{2}$-layout. We start with the biconnected case (see Section 2) and then turn towards general 4-planar graphs (see Section 3). Our upper bound of 2 for the smooth complexity of 4-planar graphs improves the previously known bound of 3 and matches the corresponding lower bound [2]. In contrast to the known algorithm for $\mathrm{SC}_{3}$-layout [2], which is based on an algorithm for $\mathrm{OC}_{3}$-layout of Biedl and Kant [3], we use an algorithm of Liu at al. [9] for $\mathrm{OC}_{3}$-layout, which avoids so-called S-shaped edges (see Figure $2 \mathrm{~b}$, top). Such edges are not desirable since they impose strong restrictions on vertex positions in a smooth orthogonal layout (see Figure 2p, bottom). Our construction requires more than polynomial area. Therefore, we made no effort in proving a bound. 
Table 1: Comparison of our results to the results of Bekos et al. [2].

\begin{tabular}{|c|c|c|c|c|}
\hline \multirow[t]{2}{*}{ graph class } & \multicolumn{3}{|c|}{ our contribution } & \multirow[t]{2}{*}{ Bekos et al. |2] } \\
\hline & complexity & area & reference & \\
\hline biconnected 4-planar & $\mathrm{SC}_{2}$ & super-poly & Theorem 3 & $\mathrm{SC}_{3}$ \\
\hline 4-planar & $\mathrm{SC}_{2}$ & super-poly & Theorem $\overline{4}$ & \\
\hline 3-planar & $\mathrm{SC}_{2}$ & $\left\lfloor n^{2} / 4\right\rfloor \times\lfloor n / 2\rfloor$ & Theorem $\overline{5}$ & \\
\hline biconnected 4-outerplane & $\mathrm{SC}_{1}$ & exponential & Theorem $\overline{6}$ & \\
\hline triconnected 3-planar & & & & $\mathrm{SC}_{1}$ \\
\hline Hamiltonian 3-planar & & & & $\mathrm{SC}_{1}$ \\
\hline poly-area & $\nsupseteq \mathrm{SC}_{1}$ & & Theorem 1 & \\
\hline $\mathrm{OC}_{3}$, octahedron & & & & $\nsubseteq \pm \mathrm{SC}_{1}$ \\
\hline $\mathrm{OC}_{2}$ & $\not \subset \mathrm{SC}_{1}$ & & Theorem 2 & \\
\hline
\end{tabular}

Further, we prove that every biconnected 4-outerplane graph admits an $\mathrm{SC}_{1}$-layout (see Section 47, expanding the class of graphs with $\mathrm{SC}_{1}$-layout from triconnected or Hamiltonian 3-planar graphs [2]. Note that in our result, the outerplane embedding can be prescribed, while in the other results the algorithms need the freedom to choose an appropriate embedding.

We complement our positive results by the following two negative results; see the appendix.

Theorem 1. There is an infinite family of graphs that require exponential area if they are drawn with $S C_{1}$.

So far, such a family of graphs has only been known under the additional, rather strong restriction of a fixed port assignment [2, Thm. 5, Fig. 7]. A port assignment prescribes, for each edge, in which direction it must enter its endpoints.

Theorem 2. There is an infinite family of biconnected 4-planar graphs that admit $\mathrm{OC}_{2}{ }^{-}$ layouts but do not admit $S C_{1}$-layouts.

So far, the only graphs known not to admit an $\mathrm{SC}_{1}$-layout were the octahedron (which is the only 4-planar graph that needs $\mathrm{OC}_{4}$ ) and a family of graphs with a fixed triangular outer face.

\section{Smooth Layouts for Biconnected 4-Planar Graphs}

In this section, we prove that any biconnected 4-planar graph admits an $\mathrm{SC}_{2}$-layout. Given a biconnected 4-planar graph, we first compute an $\mathrm{OC}_{3}$-layout, using an algorithm of Liu et al. [9]. Then we turn the result of their algorithm into an $\mathrm{SC}_{2}$-layout.

Liu et al. choose two vertices $s$ and $t$ and compute an st-ordering of the input graph. An $s t$-ordering is an ordering $(s=1,2, \ldots, n=t)$ of the vertices such that every $j$ $(2<j<n-1)$ has neighbors $i$ and $k$ with $i<j<k$. Then they draw vertices 1 and 2 on a horizontal grid line, row 1, connecting them by a so-called U-shape; see Fig. 22: They go through the other vertices as prescribed by the $s t$-ordering, placing vertex $i$ in row $i-1$. Calling an edge of which exactly one end-vertex is already drawn an open edge, they maintain the following invariant: 


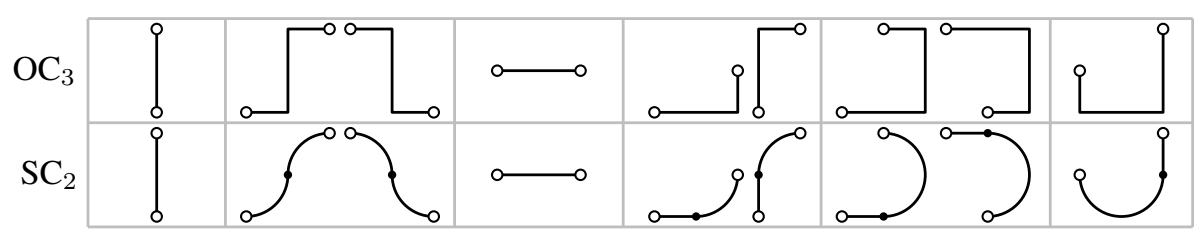
(a) vertical
(b) S-shapes
(c) horizontal
(d) L-shapes
(e) C-shapes
(f) U-shape

Fig. 2: Converting shapes from the $\mathrm{OC}_{3}$-layout to $\mathrm{SC}_{2}$.

$\left(I_{1}\right)$ In each iteration, every open edge is associated with a column (a vertical grid line).

An algorithm of Biedl and Kant [3] yields an $\mathrm{OC}_{3}$-layout similar to that of Liu et al. However, Liu et al. additionally show how to modify their algorithm such that it produces $\mathrm{OC}_{3}$-layouts without S-shapes; see Fig. $2 \mathrm{p}$ (top). In an $\mathrm{SC}_{2}$-layout, $\mathrm{S}$-shapes are composed of two quarter-circles; see Fig. $2 \mathrm{p}$ (bottom); they are undesirable as they force their endpoints to lie on a line of slope \pm 1 .

In their modified algorithm, Liu et al. search for paths in the drawing that consist only of S-shapes; every vertex lies on at most one such path. They place all vertices on such a path in the same row, without changing their column. This essentially converts all S-shapes into horizontal edges. Now every edge (except $(1,2)$ and $(1, n)$ ) is drawn as a vertical segment, horizontal segment, L-shape, or C-shape; see Fig. 2. The edge $(1,2)$ is drawn as a U-shape and the edge $(1, n)$, if it exists, is either drawn as a Cshape or (only in the case of the octahedron) as a three-bend edge that uses the left port of vertex 1 and the top port of vertex $n$.

We convert the output of the algorithm of Liu et al. from $\mathrm{OC}_{3}$ to $\mathrm{SC}_{2}$. The coordinates of the vertices and the port assignment of their drawing define a (non-planar) $\mathrm{SC}_{2}$-layout using the conversion table in Fig 3 . In order to avoid crossings, we carefully determine new vertex positions scanning the drawing of Liu et al. from bottom to top.

We now introduce our main tool for the conversion: a cut, for us, is a $y$-monotone curve consisting of horizontal, vertical, and circular segments that divides the current drawing into a left and a right part, and only intersects horizontal segments and semicircles of the drawing. In the following, we describe how one can find such a cut from any starting point at the top of the drawing; see Fig. 4 (In spite of the fact that we define the cut going from top to bottom, "to its left" will, as usually, mean "with smaller $x$ coordinate".)

When such a cut encounters a vertex $u$ to its right with an outgoing edge associated with its left port, then the cut continues by passing through the segment incident to $u$. On the other hand, if the port has an incoming L-shaped or C-shaped edge, the cut just follows the edge. The case when the cut encounters a vertex to its left is handled symmetrically.

Let $v$ be a vertex incident to two incoming C-shapes $(u, v)$ and $(w, v)$. If $y(w) \leq$ $y(u)$ we call the C-shape $(u, v)$ protected by $(w, v)$; otherwise, we call it unprotected. In order to ensure that a cut passes only through horizontal segments and that our final drawing is planar, our algorithm will maintain the following new invariants: 


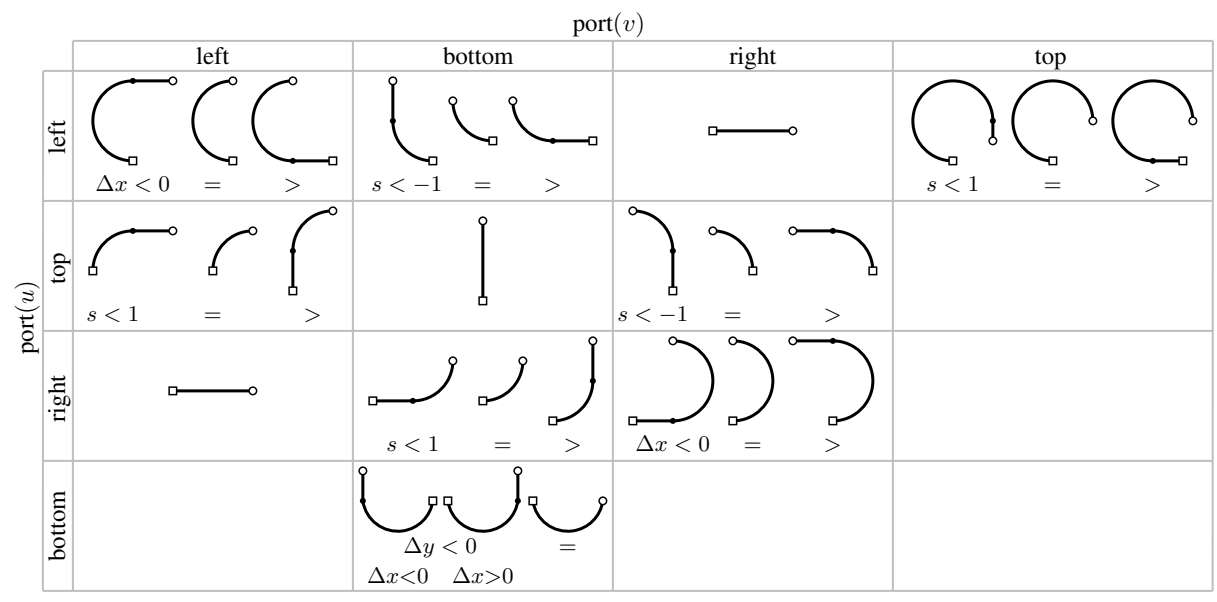

Fig. 3: Cases for drawing the edge $(u, v)$ based on the port assignment. In each case, $u$ is the lower of the two vertices $(y(u)<y(v))$. As shorthand, we use $\Delta x=x(u)-x(v), \Delta y=y(u)-y(v)$, and $s=\operatorname{slope}(u, v)=\Delta x / \Delta y$.

$\left(I_{2}\right)$ An L-shape never contains a vertical segment (as in Fig. 2d right); it always contains a horizontal segment (as in Fig. $2 \mathrm{~d}$ left) or no straight-line segment.

$\left(I_{3}\right)$ An unprotected C-shape never contains a horizontal segment incident to its top vertex (as in Fig. 2e right); it always contains a horizontal segment incident to its bottom vertex(as in Fig. 2e left) or no straight-line segment.

$\left(I_{4}\right)$ The subgraph induced by the vertices that have already been drawn has the same embedding as in the drawing of Liu et al.

Below, we treat L- and C-shapes of complexity 1 as if they had a horizontal segment of length 0 incident to their bottom vertex. Note that every cut moves around the protected C-shapes, so it will never intersect their semi-circular segments. Now we we are ready to state the main Theorem of this Section by presenting our algorithm for $\mathrm{SC}_{2}$-layouts.

Theorem 3. Every biconnected 4-planar graph admits an $\mathrm{SC}_{2}$-layout.

Proof. In the drawing $\Gamma$ of Liu et al., vertices are arranged in rows. Let $V_{1}, \ldots, V_{r}$ be the partition of the vertex set $V$ in rows $1, \ldots, r$. Following Liu et al., the vertices in each such set induce a path in $G$. We place vertices in the order $V_{1}, \ldots, V_{r}$. In this process, we maintain a planar drawing $\Gamma^{\prime}$ and the invariants $I_{1}$ to $I_{4}$. As Liu et al., we place the vertices on the integer grid. We deal with the special edges $(1,2)$ and $(1, n)$ at the end, leaving their ports, that is, the bottom and left port of vertex 1 and the top port of vertex $n$, open.

For invariant $I_{1}$, we associate each open edge with the column on which the algorithm of Liu et al. places it. If their algorithm draws the first segment of the open edge horizontally (from the source vertex to the column), we use the same segment for our drawing. We use the same ports for the edges as their algorithm. Thus, our drawing keeps the embedding of Liu et al., maintaining invariant $I_{4}$. 


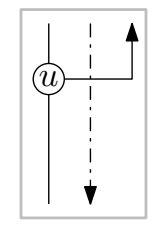

(a) open edge

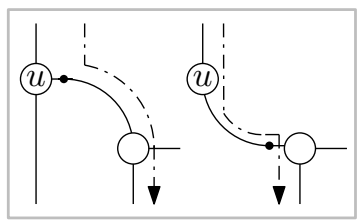

(b) L-shape

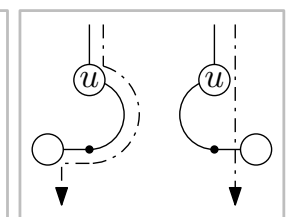

(c) C-shape

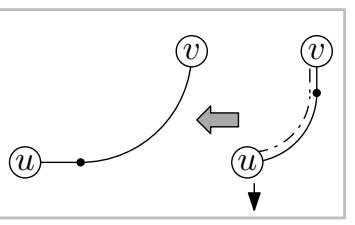

(d) Maintaining $I_{2}$

Fig. 4: Finding a cut.

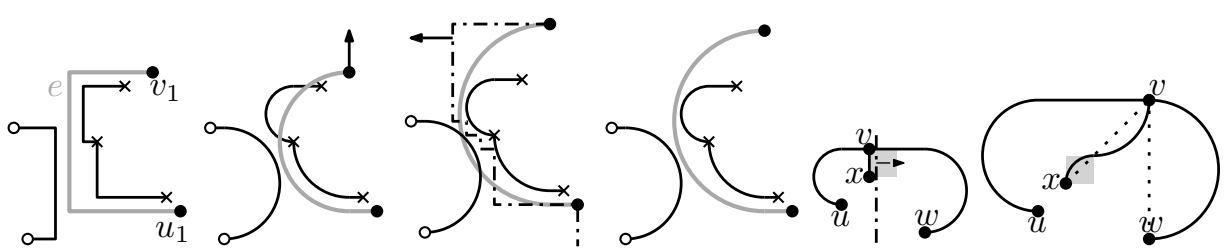

(a) $\mathrm{OC}_{3}$-layout (b) moving $v_{1}$ (c) finding a cut (d) $\mathrm{SC}_{2}$-layout $\quad$ (e)(f) protected C-shape

Fig. 5: Handling C-shapes

Assume that we have placed $V_{1}, \ldots, V_{i-1}$ and that the vertices in $V_{i}$ are $v_{1}, \ldots, v_{c}$ in left-to-right order (the case $v_{1}=v_{c}$ is possible). Vertex $v_{j}(1 \leq j \leq c$ ) is placed in the column with which the edge entering the bottom port of $v_{j}$ is associated. If the left port of $v_{1}$ is used by an incoming (L- or C-shaped) edge $e=\left(u_{1}, v_{1}\right)$, we place $v_{1}$ (and the other vertices in $V_{i}$ ) on a row high enough so that a smooth drawing of $e$ does not create any crossings with edges lying on the right side of $e$ in $\Gamma$; see Fig. 5 b.

In order to make sure that the new drawing of $e$ does not create crossings with edges on the left side of $e$ in $\Gamma$, we need to "push" those edges to the left of $e$. We do this by computing a cut that starts from $v_{1}$, separates the vertices and edges that lie on the left side of $e$ in $\Gamma$ from those on the right side, passes $u_{1}$ slightly to the left, and continues downwards as described above; see Fig. 5. Since, by invariant $I_{4}$, our drawing so far is planar and each edge is drawn in a $y$-monotone fashion, we can find a cut, too, that is $y$-monotone. We move everything on the left side of the cut further left such that $e$ has no more crossings. Note that the cut intersects only horizontal edge segments. These will simply become longer by the move.

Let $\Delta x_{i}=x\left(v_{i}\right)-x\left(u_{i}\right)$ and $\Delta y_{i}=y\left(v_{i}\right)-y\left(u_{i}\right)$ for $i=1, \ldots, c$. It is possible that the drawing of $e$ violates invariant $I_{3}$-if $u_{1}$ lies to the left of $v_{1}$. We consider two cases. First, assume that the edge $\left(u_{1}, v_{1}\right)$ is the only incoming C-shape at $v_{1}$. In this case, we simply define a cut that starts slightly to the right of $v_{1}$, follows $e$, intersects $e$ slightly to the left of $u_{1}$, and continues downwards. Then we move everything on the left side of the cut by $\Delta x_{1}+1$ units to the left. Next, assume that there is another C-shape $\left(w_{1}, v_{1}\right)$ entering the right port of $v_{1}$; see Fig. 5p. We assume w.l.o.g. that $y\left(w_{1}\right) \leq y\left(u_{1}\right)$. Let $\left(x_{1}, v_{1}\right)$ be the edge incident to the bottom vertex of $v_{1}$. In this case, we first find a cut that starts slightly to the right of $v_{1}$, follows $\left(x_{1}, v_{1}\right)$, passes $x_{1}$ slightly to the right, and continues downwards. Then we move everything on the right side of the cut by $y\left(v_{1}\right)-y\left(x_{1}\right)$ units to the right. Thus, there is an empty square to 
the right of $x_{1}$ with size $y\left(v_{1}\right)-y\left(x_{1}\right)$ - Now we place $v_{1}$ at the intersection of the diagonal through $x_{1}$ with slope 1 and the vertical line through $w_{1}$. Because of this placement, we can draw the edge $\left(x_{1}, v_{1}\right)$ by using to quarter-circles with a common horizontal tangent in the top right corner of the empty square; see Fig. 5: Note that the edge $\left(u_{1}, v_{1}\right)$ is protected by $\left(w_{1}, v_{1}\right)$, so it can have a horizontal segment incident to $v_{1}$. This establishes $I_{3}$.

It is also possible that the drawing of $e$ violates invariant $I_{2}$-if slope $\left(u_{1}, v_{1}\right)>1$. In this case we define a cut that starts slightly to the left of $v_{1}$, intersects $e$ and continues downwards. Then we move everything on the left side of the cut by $\Delta y_{1}$ units to the left. This establishes $I_{2}$.

We treat $v_{c}$, the rightmost vertex in the current row, symmetrically to $v_{1}$.

Now we have to treat the edges entering the vertices $v_{1}, \ldots, v_{c}$ from below. Note that these edges can only be vertical or L-shaped. Vertical edges can be drawn without violating the invariants. However, invariant $I_{2}$ may be violated if an edge $e_{i}=\left(u_{i}, v_{i}\right)$ entering the bottom port of vertex $v_{i}$ is L-shaped; see Fig. $4 \mathrm{~d}$. Assume that $x\left(u_{i}\right)<$ $x\left(v_{i}\right)$. In this case we find a cut that starts slightly to the left of $v_{i}$, follows $e_{i}$, intersects $e_{i}$ slightly to the right of $u_{i}$, and continues downwards. Then we move everything on the left side of the cut by $\Delta y_{i}$ units to the left. This establishes $I_{2}$. We handle the case $x\left(u_{i}\right)>x\left(v_{i}\right)$ symmetrically.

We thus place the vertices row by row and draw the incoming edges for the newly placed vertices, copying the embedding of the current subgraph from $\Gamma$. This completes the drawing of $G-\{(1,2),(1, n)\}$. Note that vertex 1 has no incoming edge and vertex 2 has only one incoming edge, that is, $(1,2)$. Thus, the bottom port of both vertices is still unused. We draw the edge $(1,2)$ as a U-shape. Finally, we finish the layout by drawing the edge $(1, n)$. By construction, the left port of vertex 1 is still unused. Note that vertex $n$ has no outgoing edges, so the top port of $n$ is still free. Hence, we can draw the edge $(1, n)$ as a horizontal or vertical segment followed by a three-quarter-circle. This completes the proof of Theorem 3 .

\section{Smooth Layouts for Arbitrary 4-Planar Graphs}

In this section, we describe how to create $\mathrm{SC}_{2}$-layouts for arbitrary 4-planar graphs. To achieve this, we decompose the graph into biconnected components, embed them separately and then connect them. For the connection it is important that one of the connector vertices lies on the outer face of its component. Within each component, the connector vertices have degree at most 3; if they have degree 2, we must make sure that their incident edges don't use opposite ports. Following Biedl and Kant [3], we say that a degree-2 vertex $v$ is drawn with right angle if the edges incident to $v$ use two neighboring ports.

Lemma 1. Any biconnected 4-planar graph admits an $S_{2}$-layout such that all degree-2 vertices are drawn with right angle.

Proof. Let $v$ be a degree-2 vertex. We now show how to adjust the algorithm of Section 2 such that $v$ is drawn with right angle. By construction, the top and the bottom ports of $v$ are used. Let $(u, v)$ be the edge entering $v$ from below (we allow $v=1$ 


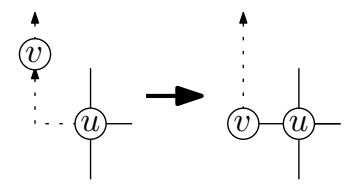

(a) an L-shape becomes a horizontal edge

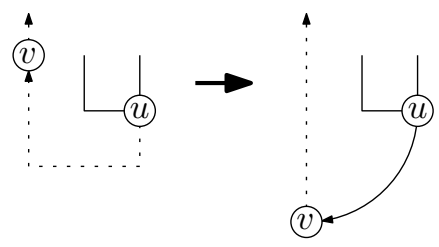

(c) a U-shape becomes an L-shape

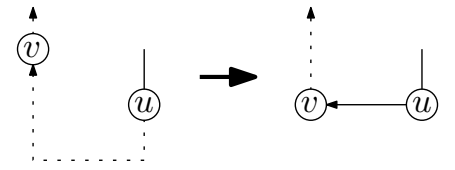

(b) a U-shape becomes a horizontal edge

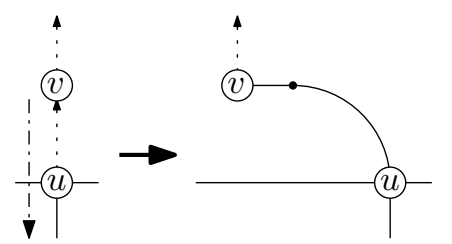

(d) a vertical edge becomes an L-shape

Fig. 6: Modification of the placement of degree-2 vertices.

and $u=2)$. We modify the algorithm such that $(u, v)$ uses the left or right rather than the bottom port of $v$. We consider three cases; $(u, v)$ is either L-shaped, $\mathrm{U}$-shaped, or vertical. These cases are handled when $v$ is drawn inserted into the smooth orthogonal drawing.

First, we assume that $(u, v)$ is L-shaped; see Fig. 6a. Then, we can simply move $v$ to the same row as $u$, making the edge horizontal.

Now, we assume that $(u, v)$ is U-shaped; see Fig. 6b. 6c. Then $u=1$ and $v=2$ or vice versa. If both have degree 2 , we move the higher vertex to the row of the lower vertex (if necessary) and replace the $\mathrm{U}$-shaped edge by a horizontal edge. Otherwise we move the vertex with degree-2, say $v$, downwards to row $y(u)-\Delta x$ such that we can replace the U-shape by an L-shape.

Otherwise, $(u, v)$ is vertical; see Fig. 6d. Then, we compute a cut that starts slightly below $v$, follows $(u, v)$ downwards, passing $u$ to its left. We move all vertices (including $u$, but not $v$ ) that lie on the right side of this cut (by at least $\Delta y$ ) to the right. Then we can draw $(u, v)$ as an L-shape that uses the right port of $v$.

Observe that, in each of the three cases, we redraw all affected edges with $\mathrm{SC}_{2}$. Hence, the modified algorithm still yields an $\mathrm{SC}_{2}$-layout. At the same time, all degree- 2 vertices are drawn with right angle as desired.

Now we describe how to connect the biconnected components. Recall that a bridge is an edge whose removal disconnects a graph $G$. We call the two endpoints of a bridge bridge heads. A cut vertex is a vertex whose removal disconnects the graph, but is not a bridge head.

Theorem 4. Any 4-planar graph admits an $\mathrm{SC}_{2}$-layout.

Proof. Let $G_{0}$ be some biconnected component of $G$, and let $v_{1}, \ldots, v_{k}$ be the cut vertices and bridge heads of $G$ in $G_{0}$. For $i=1, \ldots, k$, if $v_{i}$ is a bridge head, let $v_{i}^{\prime}$ be the other head of the bridge, otherwise let $v_{i}^{\prime}=v_{i}$. Let $G_{i}$ be the subgraph of $G$ 
containing $v_{i}^{\prime}$ and the connected components of $G-v_{i}^{\prime}$ not containing $G_{0}$. Following Lemma 1. $G_{0}$ can be drawn such that all degree-2 vertices are drawn with right angles.

The algorithm of Section 2 that we modified in the proof of Lemma 1 places the last vertex $(n)$ at the top of the drawing and thus on the outer face. When drawing $G_{i}$, we choose $v_{i}^{\prime}$ as this vertex. By induction, $G_{i}$ can be drawn such that all degree- 2 vertices are drawn with right angles.

In order to connect $G_{i}$ to $G_{0}$, we make $G_{0}$ large enough to fit $G_{i}$ into the face that contains the free ports of $v_{i}$. We may have to rotate $G_{i}$ by a multiple of $90^{\circ}$ to achieve the following. If $v_{i}$ is a cut vertex, we make sure that $v_{i}^{\prime}$ uses the ports of $v_{i}$ that are free in $G_{0}$. Then we identify $v_{i}$ and $v_{i}^{\prime}$. Otherwise we make sure that a free port of $v_{i}$ and a free port of $v_{i}^{\prime}$ are opposite. Then we draw the bridge $\left(v_{i}, v_{i}^{\prime}\right)$ horizontally or vertically. This completes our proof.

For an example run of our algorithm, see Fig. 15 in Appendix C. For graphs of maximum degree 3 , we can make our drawings more compact. This is due to the fact that we can avoid C-shaped edges (and hence cuts) completely. In the presence of Lshapes only, it suffices to stretch the orthogonal drawing by a factor of $n$.

Theorem 5. Every biconnected 3-planar graph with $n$ vertices admits an $S_{2}$-layout using area $\left\lfloor n^{2} / 4\right\rfloor \times\lfloor n / 2\rfloor$.

Proof. It is known that every biconnected 3-planar graph except $K_{4}$ has an $\mathrm{OC}_{2}$-layout using area $\lfloor n / 2\rfloor \times\lfloor n / 2\rfloor$ from Kant [7]. Now we use the same global stretching as Bekos et al. when they showed that every $\mathrm{OC}_{2}$-layout can be transformed into an $\mathrm{SC}_{2}$-layout [2, Thm. 2]: we stretch the drawing horizontally by the height of the drawing, that is, by a factor of $\lfloor n / 2\rfloor$. This makes sure that we can replace every bend by a quarter circle without introducing crossings. Figure 7 shows an $\mathrm{SC}_{1}$-layout of $K_{4}$; completing our proof.

\section{$4 \mathrm{SC}_{1}$-Layouts of Biconnected 4-Outerplane Graphs}

In this section, we consider 4-outerplane graphs, that is, 4-outerplanar graphs with an outerplanar embedding. We prove that any biconnected 4-outerplane graph admits an $\mathrm{SC}_{1}$-layout. To do so, we first prove the result for a subclass of 4-outerplane graphs, which we call $(2,3)$-restricted outerplane graphs; then we generalize. Recall that the weak dual of a plane graph is the subgraph of the dual graph whose vertices correspond to the bounded faces of the primal graph.

Definition 1. A 4-outerplane graph is called $(2,3)$-restricted if it contains a pair of consecutive vertices on the outer face, $x$ and $y$, such that $\operatorname{deg}(x)=2$ and $\operatorname{deg}(y) \leq 3$.

Lemma 2. Any biconnected (2,3)-restricted 4-outerplane graph admits an $S C_{1}$-layout.

Proof. Let $x$ and $y$ be two vertices, consecutive on the outer face of the given graph $G$ such that $\operatorname{deg}(x)=2$ and $\operatorname{deg}(y) \leq 3$. Let also $T$ be the weak dual tree of $G$ rooted at the node, say $v^{*}$, of $T$ corresponding to the bounded face, say $f^{*}$, containing both $x$ 
and $y$. We construct the $\mathrm{SC}_{1}$-layout $\Gamma$ for $G$ by traversing $T$, starting with $v^{*}$. When we traverse a node of $T$, we draw the corresponding face of $G$ with $\mathrm{SC}_{1}$.

Consider the case when we have constructed a drawing $\Gamma(H)$ for a connected subgraph $H$ of $G$ and we want to add a new face $f$ to $\Gamma(H)$. For each vertex $u$ of $H$, let $p_{u}=(x(u), y(u))$ denote the point at which $u$ is drawn in $\Gamma(H)$. The remaining degree of $u$ is the number of vertices adjacent to $u$ in $G-H$. Since we construct $\Gamma(H)$ face by face, the remaining degree of each vertex in $H$ is at most two. The free ports of $u$ are the ones that are not occupied by an edge of $H$ in $\Gamma(H)$. During the construction of $\Gamma$, we maintain the following four invariants:

$\left(J_{1}\right) \Gamma(H)$ is an $\mathrm{SC}_{1}$-layout that preserves the planar embedding of $G$, and each edge is drawn either as an axis-parallel line segment or as a quarter-circle in $\Gamma(H)$. (Note that we do not use semi- and 3/4-circles.)

$\left(J_{2}\right)$ For each vertex $u$ of $H$, the free ports of $u$ in $\Gamma(H)$ are consecutive around $u$, and they point to the outer face of $\Gamma(H)$.

$\left(J_{3}\right)$ Vertices with remaining degree exactly 2 are incident to an edge drawn as a quarter-circle.

$\left(J_{4}\right)$ If an edge $(u, v)$ is drawn as an axis-parallel segment, then at least one of $u$ and $v$ has remaining degree at most 1 . If $(u, v)$ is vertical and $y(u)<y(v)$, then $u$ has remaining degree at most 1 and the free port of $u$ in $\Gamma(H)$ is horizontal; see Figs. $8 \mathrm{a}, 8 \mathrm{~d}$ and $8 \mathrm{~g}$. Symmetrically, if $(u, v)$ is horizontal and $x(u)<x(v)$, then $u$ has remaining degree at most 1 and the free port of $u$ in $\Gamma(H)$ is vertical; see Figs. 8 p, 8 e and 8 h.

We now show how we add the drawing of the new face $f$ to $\Gamma(H)$. Since $G$ is biconnected and outerplanar, and due to the order in which we process the faces of $G$, $f$ has exactly two vertices, say $u$ and $v$, which have already been drawn (as $p_{u}$ and $p_{v}$ ). The two vertices are adjacent. Depending on how the edge $(u, v)$ is drawn in $\Gamma(H)$, we draw the remaining vertices and edges of $f$.

Let $k \geq 3$ be the number of vertices on the boundary of $f$. The slopes of the line segment $\overline{p_{u} p_{v}}$ is in $\{-1,0,+1, \infty\}$, where $\infty$ means that $\overline{p_{u} p_{v}}$ is vertical. For $s \in\{-1,0,+1, \infty\}$, we denote by $\ell_{u}^{s}$ the line with slope $s$ through $p_{u}$. Similarly, we denote by $\ell_{u, \varepsilon}^{s}$ the line with slope $s$ through the point $(x(u)+\varepsilon, y(u))$, for some $\varepsilon>0$. Figs. $8 \mathrm{~d}-8$ f show the drawing of $f$ for $k=3$, and Figs. 8 - 8 , for any $k \geq 4$.

Note that the lengths of the line segments and the radii of the quarter-circles that form $f$ are equal (except for the radii of the bold-drawn quarter-circles of Figs. $8 \mathrm{~g}$ and $8 \mathrm{~h}$ which are determined by the remaining edges of $f$ ). Hence, the lengths of the line segments and the radii of the quarter-circles that form any face that is descendant of face $f$ in $T$ are smaller than or equal to the lengths of the line segments and the radii of the quarter-circles that form $f$. Our construction ensures that all vertices of the subgraph of $G$ induced by the subtree of $T$ rooted at $f$ lie in the interior or on the boundary of the diagonal semi-strip $L_{u v}$ delimited by $\ell_{u}^{+1}, \ell_{v}^{+1}$, and $\overline{p_{u} p_{v}}$ (see Fig. $8 \mathrm{k}$ ). The only edges of this subgraph that are drawn in the complement of $L_{u v}$ (and are potentially involved in crossings) are incident to two vertices that both lie on the boundary of $L_{u v}$. In this particular case, however, the degree restriction implies that $L_{u v}$ is surrounded from above and/or below by two empty diagonal semi-strips of at least half the width of 


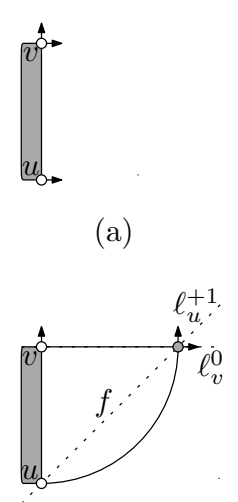

(d)

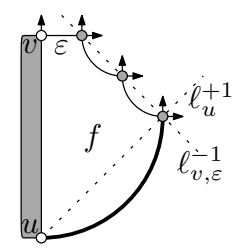

(g)

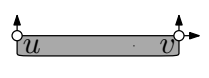

(b)

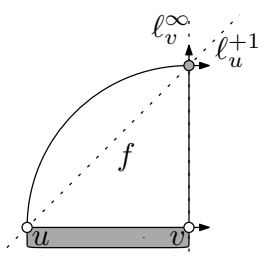

(e)

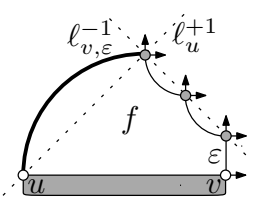

(h)

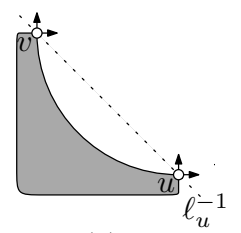

(c)

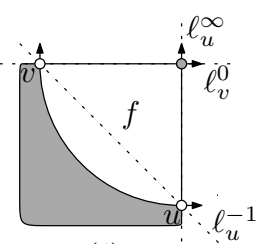

(f)

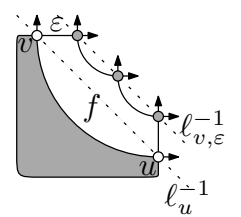

(i)

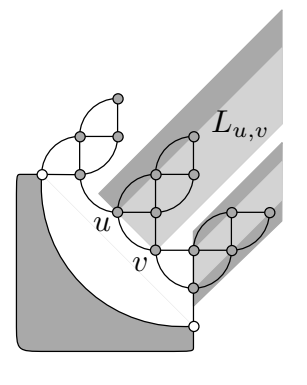

$(\mathrm{k})$

Fig. 8: (a)-(i) Different cases that arise when drawing face $f$ of $G$. (k) A sample drawing.

semi-strip $L_{u v}$, which is enough to ensure planarity for two reasons. First, any face that is descendant of face $f$ in $T$ is formed by line segments and quarter-circles of radius that are at most as big as the corresponding ones of face $f$. Second, due to the degree restrictions, if two neighboring children of $f$ are triangles, the left one cannot have a right child and vice versa.

Let us summarize. Fig. $8 \mathrm{~d}-8$, show that the drawing of $f$ ensures that invariants $(J \overline{1})-$ ( 哽) of our algorithm are satisfied for $H \cup\{f\}$. We begin by drawing the root face $f^{*}$. Since $G$ is $(2,3)$-restricted, $f^{*}$ has two vertices $x$ and $y$ consecutive on the outer face with $\operatorname{deg}(x)=2$ and $\operatorname{deg}(y) \leq 3$. We draw edge $(x, y)$ as a vertical line segment. Then the remaining degrees of $x$ and $y$ are 1 and 2, respectively, which satisfies the invariants for face $f^{*}$. Hence, we complete the drawing of $f^{*}$ as in Fig. $8 \mathrm{~d}$ or $8 \mathrm{~g}$. Traversing $T$ in pre-order, we complete the drawing of $G$.

Next, we show how to deal with general biconnected 4-outerplane graphs. Suppose $G$ is not $(2,3)$-restricted. As the following lemma asserts, we can always construct a biconnected $(2,3)$-restricted 4-outerplane graph by deleting a vertex of degree 2 from $G$.

Lemma 3. Let $G=(V, E)$ be a biconnected 4-outerplane graph that is not $(2,3)$ restricted. Then $G$ has a degree-2 vertex whose removal yields a $(2,3)$-restricted biconnected 4-outerplane graph. 
Proof. The proof is by induction on the number of vertices. The base case is a maximal biconnected outerplane graph on six vertices, which is the only non-(2,3)-restricted graph with six or less vertices. It is easy to see that in this case the removal of any degree-2 vertex yields a biconnected $(2,3)$-restricted 4-outerplane graph. Now assume that the hypothesis holds for any biconnected 4-outerplane graph with $k \geq 6$ vertices. Let $G_{k+1}$ be a biconnected 4-outerplane graph on $k+1$ vertices, which is not $(2,3)$ restricted. Let $\mathcal{F}$ be a face of $G_{k+1}$ that is a leaf in its weak dual. Then $\mathcal{F}$ contains only one internal edge and exactly two external edges since, if it contained more than two external edges, $G_{k+1}$ would be $(2,3)$-restricted. Therefore, $\mathcal{F}$ consists of three vertices, say $a, b$ and $c$, consecutive on the outer face and $\operatorname{deg}(a)=\operatorname{deg}(c)=4$, since otherwise $G_{k+1}$ would be $(2,3)$-restricted. By removing $b$, we obtain a new graph, say $G_{k}$, on $k$ vertices. If $a$ or $c$ is incident to a degree-2 vertex in $G_{k}$, then $G_{k}$ is $(2,3)$-restricted. Otherwise, by our induction hypothesis, $G_{k}$ has a degree- 2 vertex whose removal yields a $(2,3)$-restricted outerplanar graph. Since this vertex is neither adjacent to $a$ nor $c$, the removal of this vertex makes $G_{k+1}$, too, $(2,3)$-restricted.

Theorem 6. Any biconnected 4-outerplane graph admits an $S C_{1}$-layout.

Proof. If the given graph $G$ is $(2,3)$-restricted, then the result follows from Lemma 2 Thus, assume that $G$ is not $(2,3)$-restricted. Then, $G$ contains a degree-2 vertex, say $b$, whose removal yields a biconnected $(2,3)$-restricted 4-outerplane graph, say $G^{\prime}$. Hence, we can apply the algorithm of Lemma 2 to $G^{\prime}$ and obtain an outerplanar $\mathrm{SC}_{1}$-layout $\Gamma\left(G^{\prime}\right)$ of $G^{\prime}$. Since this algorithm always maintains consecutive free ports for each vertex and the neighbors of $b$ are on the outer face of $\Gamma\left(G^{\prime}\right)$, we insert insert $b$ and its two incident edges to obtain an $\mathrm{SC}_{1}$-layout $\Gamma(G)$ of $G$ as follows. Let $a$ and $c$ be the neighbors of $b$ and assume w.l.o.g. that $c$ is drawn above $a$. If edge $(a, c)$ is drawn as a quarter-circle, then a 3/4-circle arc from $p_{c}$ to $p_{b}$ and a quarter-circle from $p_{b}$ to $p_{a}$ suffice. Otherwise, line segment $\overline{p_{a} p_{b}}$ and a quarter-circle from $p_{b}$ to $p_{c}$ do the job.

\section{Conclusions and Open Problems}

In this paper, we presented several new results about smooth orthogonal drawings of 4-planar graphs. Many problems remain open, for example:

1. Can all 4-planar graphs be drawn in polynomial area with $\mathrm{SC}_{2}$ ? We have shown this only for 3-planar graphs.

2. Identify larger classes of graphs admitting $\mathrm{SC}_{1}$-layouts, e.g., do all (not necessarily biconnected) 4-outerplanar or all 3-planar graphs admit $\mathrm{SC}_{1}$-layouts?

3. We strongly conjecture that it is NP-hard to decide whether a 4-planar graph has an $\mathrm{SC}_{1}$-layout, but we struggled with some details in our attempt for a proof.

\section{References}

1. Clipping of the public transport map Gmunden - Vöcklabruck - Salzkammergut, austria. www.ooevv.at/uploads/media/OOE2_Salzkammergut_V17_END.pdf. 
2. M. A. Bekos, M. Kaufmann, S. G. Kobourov, and A. Symvonis. Smooth orthogonal layouts. In Proc. 20th Int. Symp. Graph Drawing (GD’12), pages 150-161. Springer, 2013.

3. T. Biedl and G. Kant. A better heuristic for orthogonal graph drawings. Comput. Geom. Theory Appl., 9(3):159-180, 1998.

4. C. A. Duncan, D. Eppstein, M. T. Goodrich, S. G. Kobourov, and M. Nöllenburg. Lombardi drawings of graphs. J. Graph Algorithms Appl., 16(1):85-108, 2012.

5. C. A. Duncan, D. Eppstein, M. T. Goodrich, S. G. Kobourov, and M. Nöllenburg. Drawing trees with perfect angular resolution and polynomial area. Discrete Comput. Geom., 49(2):157-182, 2013.

6. A. Garg and R. Tamassia. On the computational complexity of upward and rectilinear planarity testing. SIAM J. Comput., 31(2):601-625, 2001.

7. G. Kant. Drawing planar graphs using the canonical ordering. Algorithmica, 16(1):4-32, 1996.

8. C. E. Leiserson. Area-efficient graph layouts (for VLSI). In Proc. 21st Annu. IEEE Symp. Foundat. Comput. Sci. (FOCS'80), pages 270-281, 1980.

9. Y. Liu, A. Morgana, and B. Simeone. A linear algorithm for 2-bend embeddings of planar graphs in the two-dimensional grid. Discrete Appl. Math., 81(1-3):69-91, 1998.

10. J. Seemann. Extending the Sugiyama algorithm for drawing UML class diagrams: Towards automatic layout of object-oriented software diagrams. In G. Di Battista, editor, Proc. 5th Int. Symp. Graph Drawing (GD’97), volume 1353 of LNCS, pages 415-424. Springer, 1997.

11. R. Tamassia. On embedding a graph in the grid with the minimum number of bends. SIAM J. Comput., 16(3):421-444, 1987. 


\section{A A Lower Bound for the Area Requirement of $\mathrm{SC}_{1}$-Layouts}

In this section, we demonstrate an infinite family of 4-planar graphs that require exponential area if they are drawn with $\mathrm{SC}_{1}$. Bekos et al. [2] presented such a family of graphs for the rather restricted setting where both the embedding of the graph and the port assignment of the edges are fixed. Here, we strengthen this result. Consider the graph shown in Fig. 9a. This graph consists of several layers. Each layer consists of a cycle of four pairs of adjacent triangles. The $\mathrm{SC}_{1}$-layout of this graph in Fig. $9 \mathrm{~b}$ obviously requires exponential area since every layer uses more than twice the area of the previous layer. We will now show that this is the only $\mathrm{SC}_{1}$-layout of the graph, up to translation, rotation and scaling.

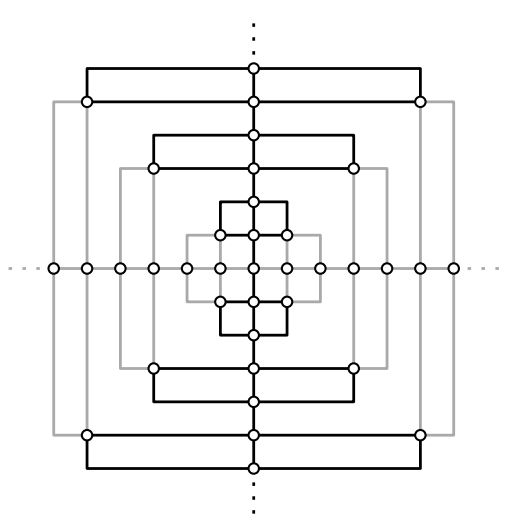

(a)

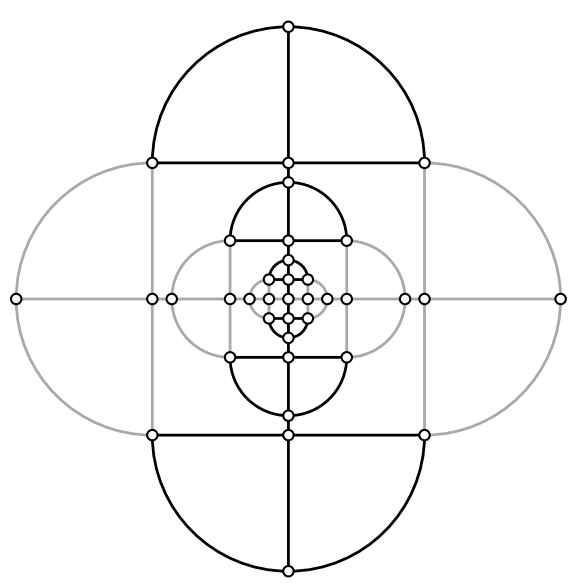

(b)

Fig. 9: (a) A graph with an $\mathrm{OC}_{2}$-layout using polynomial area (left) and (b) an $\mathrm{SC}_{1}$-layout using exponential area (right).

First, we show that there are only two ways to draw one of the triangles of each layer. In Fig. 10, we show all 16 possible ways to get an $\mathrm{SC}_{1}$-layout of a triangle. However, in our graph all free ports have to lie on the outer face. There are only two $\mathrm{SC}_{1}$-layouts of a triangle that have this property, marked by a dashed circle.

Next, we build a pair of adjacent triangles. In Fig. 11, we show that there are three ways to combine two triangles that share an edge. Finally, we combine four pairs of adjacent triangles to one layer of the graph. Using careful case analysis, it can be shown that there are only two ways to draw one of the layers with $\mathrm{SC}_{1}$; see Fig. 12 However, it is easy to see that it is impossible to connect the drawing shown in Fig. $12 \mathrm{c}$ to another layer. Thus, the $\mathrm{SC}_{1}$-layout shown in Fig. $9 \mathrm{~b}$ is the only way to draw this graph, which proves the following theorem.

Theorem 1. There is an infinite family of graphs that require exponential area if they are drawn with $S C_{1}$. 

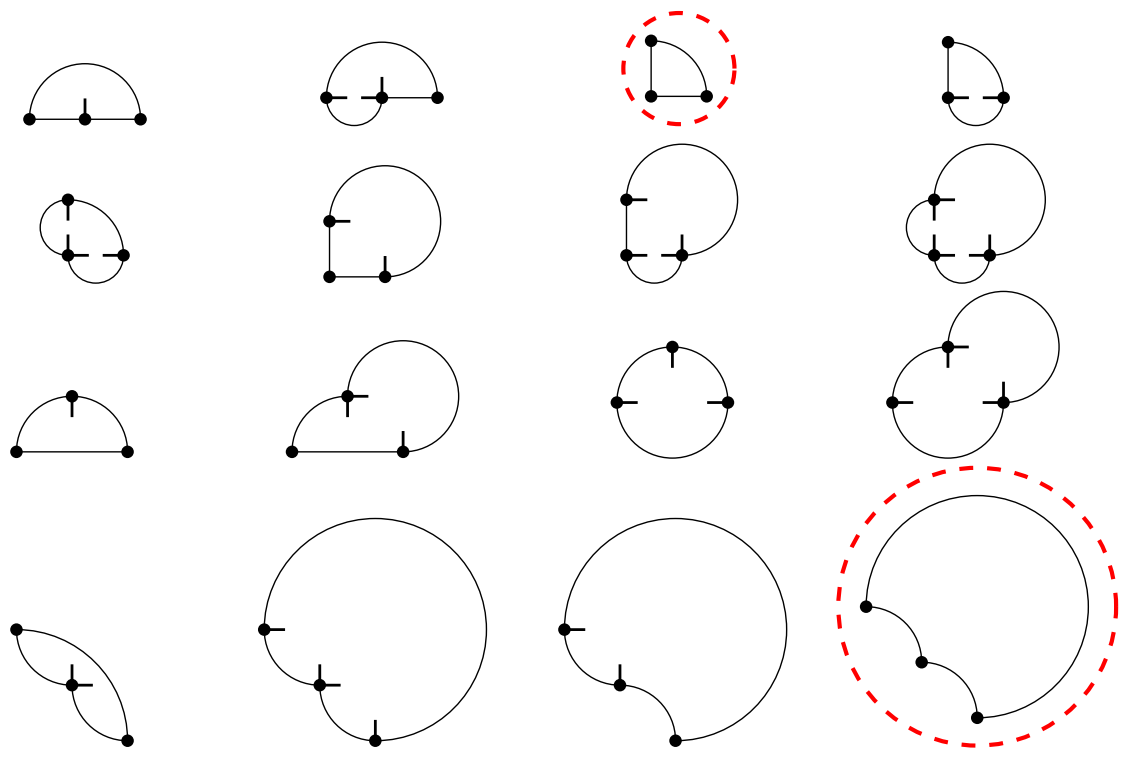

Fig. 10: All possible ways to get an $\mathrm{SC}_{1}$-layout of a triangle. Only two of these drawings (enclosed by dashed red circles) have all their ports on the outer face.
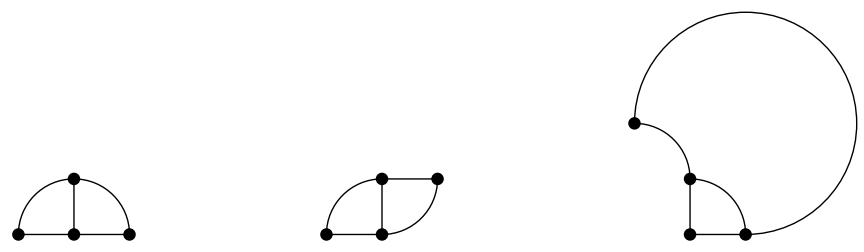

Fig. 11: There are three ways to draw two adjacent triangles.
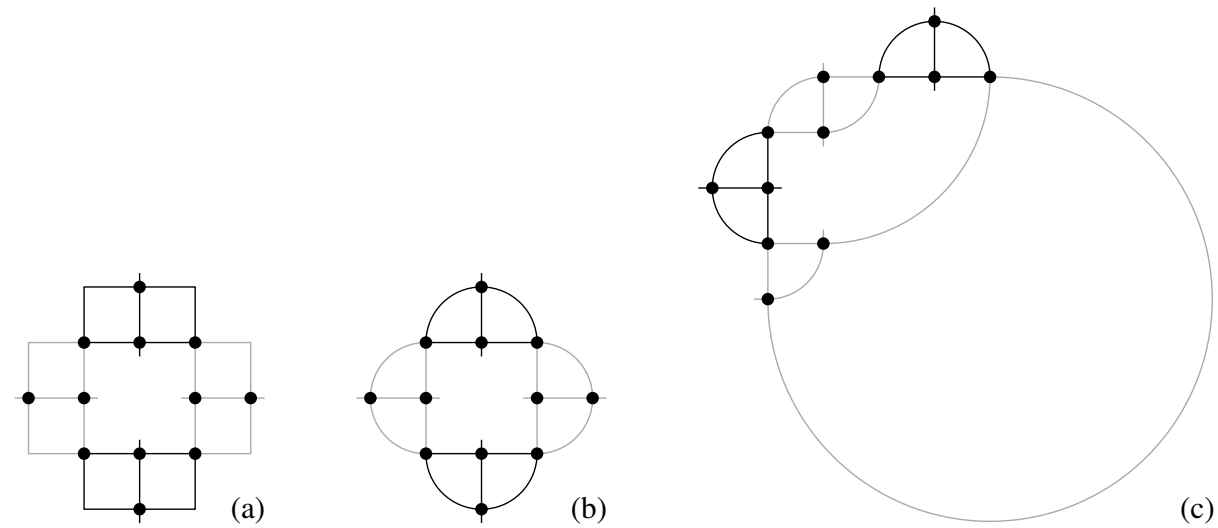

Fig. 12: (a) One layer of the graph in Fig. 9 (b) \& (c) The only two ways to draw the subgraph depicted in (a) with $\mathrm{SC}_{1}$. 


\section{B Biconnected Graphs without $\mathrm{SC}_{1}$-Layouts}

In this section, we demonstrate an infinite family of biconnected 4-planar graphs that admit $\mathrm{OC}_{2}$-layouts, but do not admit $\mathrm{SC}_{1}$-layouts. Bekos et al. [2] presented such a family of graphs assuming a rather restricted setting in which the choice of the outerface is fixed and always corresponds to a triangle. Here, we strengthen this results by providing an infinite family of biconnected 4-planar graphs that admit no $\mathrm{SC}_{1}$-layout in any embedding. We start with the following lemma.

Lemma 4. There exists a biconnected 4-planar graph that admits an $\mathrm{OC}_{2}$-layout, but does not admit an $\mathrm{SC}_{1}$-layout.

Proof. Let $G$ be the graph of Fig. $13 \mathrm{a}$. We prove that $G$ has no $\mathrm{SC}_{1}$-layout. First, note that $G$ contains two copies of the graph depicted in Fig. 13b We denote this graph by $H$. We first prove that $H$ has no $\mathrm{SC}_{1}$-layout with the given embedding. In particular, we show that the subgraph of $H$ induced by the vertices on or inside the black cycle cannot be drawn with $\mathrm{SC}_{1}$.

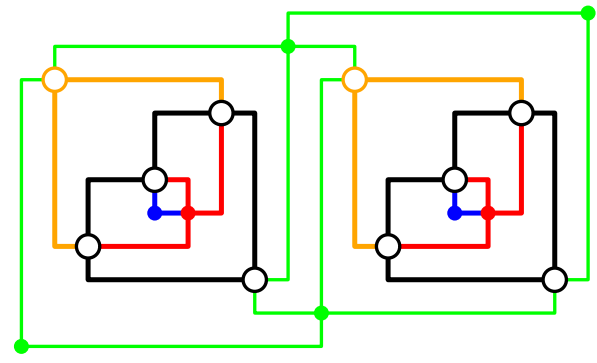

(a)

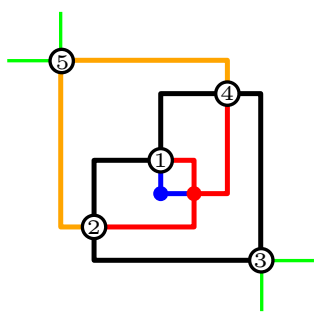

(b)

Fig. 13: (a) A graph that admits an $\mathrm{OC}_{2}$-layout, but does not admit an $\mathrm{SC}_{1}$-layout; (b) the important part of (a) in detail.

Consider edge $e=(1,2)$ of $H$. This edge can be drawn as a straight-line segment, quarter circle, semi-circle or 3/4-circle. Fig. 14 illustrates the case where $e$ is drawn as a horizontal line segment. In this case, the ports for the edges are fixed due to the given embedding and it is not possible to complete the drawing. The case where $e$ is drawn as a vertical segment is analogous. Similarly, we show that there is no $\mathrm{SC}_{1}$-layout for $H$ if $e$ is drawn as a quarter-circle in Figs. $14 \mathrm{p}-14 \mathrm{k}$, as a semi-circle in Figs. $14 \mathrm{~d}-14 \mathrm{~g}$ and as a 3/4-circle in Figs. 14h-14. Thus, there is no $\mathrm{SC}_{1}$-layout for this fixed embedding of $H$.

Next, we claim that there is no $\mathrm{SC}_{1}$-layout for any embedding of $H$ where the vertices 2, 3, 4, and 5 define the outer cycle. Indeed, if the outerface is fixed, then the only way to find a different embedding is to find a separating pair $\{u, v\}$ in $H$ and "flip" one of the components of $H-\{u, v\}$. There are two possible separating pairs in $H$ : (i) vertex 1 and the red vertex; then the flip with respect to this pair gives an isomorphic 


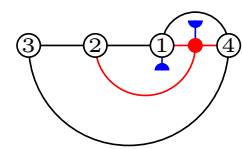

(a)

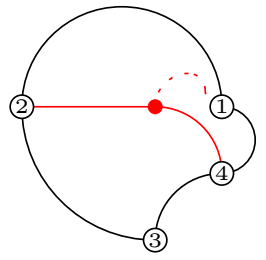

(d)

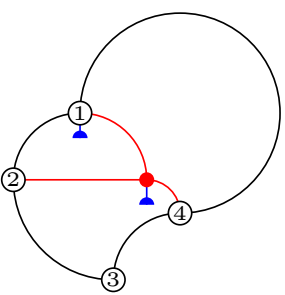

(b)

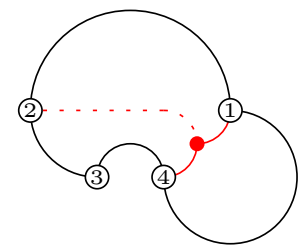

(e)

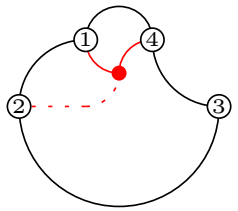

(c)

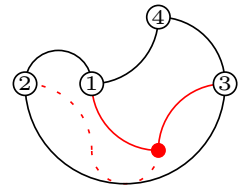

(f)

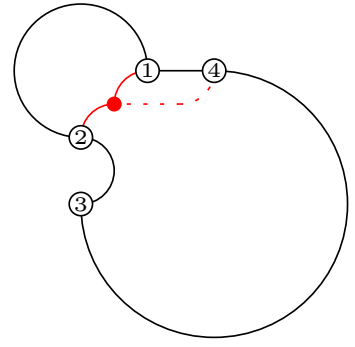

(i)

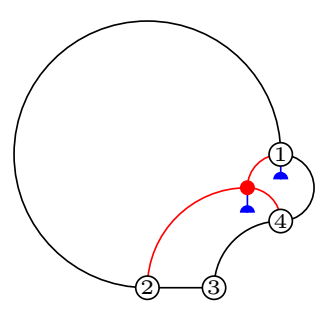

(j)

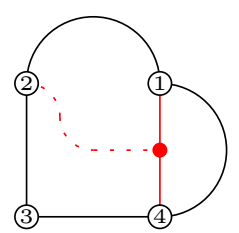

(d)

(h)

Fig. 14: Illustration for the proof of Lemma 4

graph due to symmetry; and (ii) vertices 2 and 4; then the flip with respect to this pair again gives an isomorphic graph by interchanging the role of 3 and 5. Thus, with the fixed outer cycle $(2,3,4,5)$, all possible embeddings of $H$ are isomorphic. Since $G$ contains two copies of $H$, in any embedding of $G$, at least one of the copies will retain its outer cycle. Hence, there is no $\mathrm{SC}_{1}$-drawing for any embedding of $G$.

Graph $G$ of Fig. 13a uses a few short paths to connect two copies of $H$. Obviously, we can add an arbitrary number of vertices to these paths such that the augmented graph remains biconnected and 4-planar. This proves the following theorem.

Theorem 2. There is an infinite family of biconnected 4-planar graphs that admit $O C_{2}$ layouts but do not admit $S C_{1}$-layouts. 


\section{An Example Run of Our Algorithm for $\mathrm{SC}_{2}$-Layout}

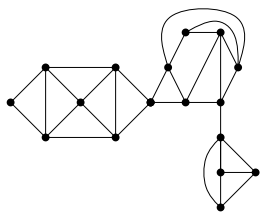

(a) input graph
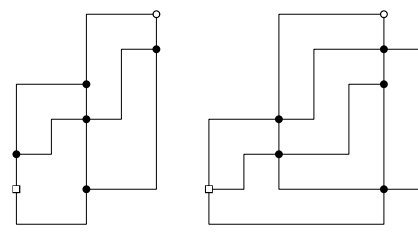

(c) st-ordering
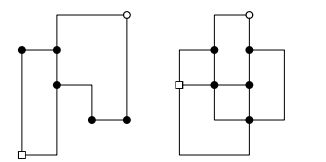

(e) drawing degree-2 vertices with right angle
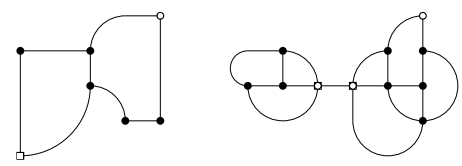

(g) connecting second and fourth part by using the bridge (third part)

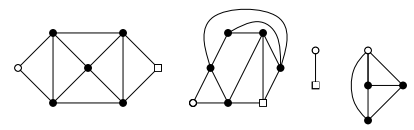

(b) biconnected subgraphs

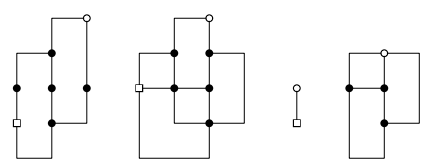

(d) eliminating S-shapes

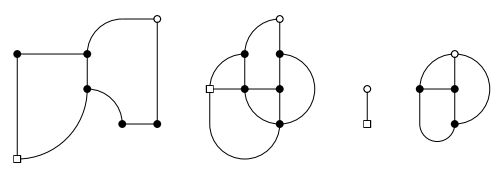

(f) drawing subgraphs with $\mathrm{SC}_{2}$

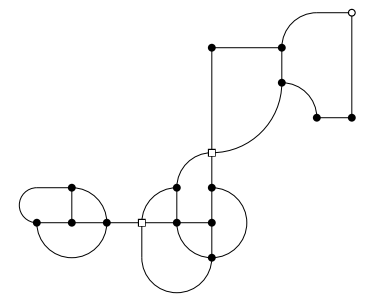

(h) connecting first and second part

Fig. 15: An example-run of our Algorithm for $\mathrm{SC}_{2}$-layout. The circle vertices of component $i$ correspond to the cut vertex $v_{i}^{\prime}$. The square vertices correspond to cut vertices of other components.

Acknowledgments. We thank Therese Biedl for pointers to Figure 1 and the work of Liu et al. [9]. 